\title{
Neural correlates of executive function and working memory in the 'at-risk mental state'
}

Matthew R. Broome, Pall Matthiasson, Paolo Fusar-Poli, James B. Woolley, Louise C. Johns, Paul Tabraham, Elvira Bramon, Lucia Valmaggia, Steven C. R. Williams, Michael J. Brammer, Xavier Chitnis and Philip K. McGuire

\section{Background}

People with prodromal symptoms have a very high risk of developing psychosis.

\author{
Aims \\ To use functional magnetic resonance imaging to examine \\ the neurocognitive basis of this vulnerability.
}

\section{Method}

Cross-sectional comparison of regional activation in individuals with an 'at-risk mental state' (at-risk group: $n=17$ ), patients with first-episode schizophreniform psychosis (psychosis group: $n=10$ ) and healthy volunteers (controls: $n=15)$ during an overt verbal fluency task and an $N$-back working memory task.

\section{Results}

A similar pattern of between-group differences in activation was evident across both tasks. Activation in the at-risk group was intermediate relative to that in controls and the psychosis group in the inferior frontal and anterior cingulate cortex during the verbal fluency task and in the inferior frontal, dorsolateral prefrontal and parietal cortex during the $N$-back task.

\section{Conclusions}

The at-risk mental state is associated with abnormalities of regional brain function that are qualitatively similar to, but less severe than, those in patients who have recently presented with psychosis

\section{Declaration of interest}

None. Funding detailed in Acknowledgements.
People with prodromal symptoms of psychosis have a $25-40 \%$ risk of developing a psychotic disorder in the following 12 months ${ }^{1,2}$ and thus have an 'at-risk mental state'. Neuropsychological studies indicate people with an at-risk mental state show impairments in executive and memory functions, with performance often intermediate between that in patients with schizophrenia and controls ${ }^{3}$ and with working memory performance predicting the onset of psychosis. ${ }^{4}$ Structural magnetic resonance imaging (MRI) studies suggest that the at-risk mental state is associated with reduced grey matter volume in regions that are also abnormal in schizophrenia, ${ }^{5}$ and a recent functional MRI study reported differential prefrontal activation in individuals with an at-risk mental state relative to controls and patients with schizophrenia during a visual oddball paradigm. ${ }^{6}$ Taken together, these findings suggest that individuals with an at-risk mental state display neurocognitive abnormalities that are qualitatively similar to, but less severe than, those seen in schizophrenia. We tested this hypothesis using functional MRI in conjunction with classic tasks of executive function and working memory.

\section{Methods}

\section{Participants}

At-risk mental state (at-risk) group ( $n=17)$

Individuals meeting Personal Assessment and Crisis Evaluation ${ }^{7}$ (PACE) criteria for an at-risk mental state were recruited from Outreach and Support in South London (OASIS). ${ }^{8}$ The diagnosis was based on assessment by two experienced clinicians using the comprehensive assessment for the at-risk mental state, ${ }^{2}$ and a consensus meeting with the clinical team. None of the participants had ever received antipsychotic medication. Briefly, an individual meets PACE criteria for an at-risk mental state if they display one or more of the following: 'attenuated' positive symptoms; frank psychotic symptoms that last less than 1 week and resolve without treatment; a recent decline in function coupled with either schizotypal personality disorder or a first-degree relative with psychosis. The individuals recruited were representative of the local population of people presenting with an at-risk mental state in terms of age, gender, ethnicity, and duration and intensity of symptoms. ${ }^{8}$

\section{First-episode (psychosis) group $(n=10)$}

Participants were patients who had presented with a first episode of psychosis to Lambeth Early Onset Services. All met ICD-10 9 criteria for a schizophreniform psychosis at the time of scanning and subsequently met the Operationalized Criteria (OPCRIT) ${ }^{10}$ threshold for a diagnosis of schizophrenia when reassessed 12 months after first presentation. Three patients were medication naive. Seven had been treated with either oral risperidone or quetiapine for a mean of 10 days (95\% CI 4.7-16.3) at mean doses of $1.7 \mathrm{mg}$ and $63.75 \mathrm{mg}$ respectively. Patients were scanned as soon after presentation as was practicable, and all but one were scanned within 2 weeks of presentation.

\section{Control group $(n=15)$}

Healthy volunteers were recruited via advertisements in the local media.

All individuals lived in the same borough of London as the clinical participants (Lambeth), were native speakers of English and were right-handed.

Individuals were excluded if there was a history of neurological disorder or they met DSM-IV ${ }^{11}$ criteria for a substance misuse disorder. General intellectual function was estimated in all participants using the National Adult Reading Test. ${ }^{12}$ The severity of symptoms in the clinical groups was assessed with the Positive and Negative Syndrome Scale (PANSS) ${ }^{13}$ on the day 
of scanning. Additionally, individuals were excluded from the analysis after data collection if they were unable to perform the cognitive tasks during image acquisition as detailed below. For the at-risk group, 19 participants underwent functional MRI, with 2 being excluded because they did not perform the task, resulting in $n=17 ; 1$ participant was excluded from the psychosis group, and one from the control group, leaving data being reported for $n=10$ and $n=15$ respectively.

There were no significant group differences in sociodemographic variables or IQ. Both positive and general PANSS scores were higher in the psychosis group than in the at-risk group, but these differences were not significant (Table 1).

\section{Image acquisition}

Images were acquired on a $1.5 \mathrm{~T}$ Signa (GE) system at the Maudsley Hospital, London; $\mathrm{T}_{2}{ }^{*}$-weighted images were acquired with a repetition time (TR) of $2 \mathrm{~s}, 38 \times 3 \mathrm{~mm}$ slices, with a $0.3 \mathrm{~mm}$ gap, in 14 axial planes. During the verbal fluency task a gradient-echo sequence $(\mathrm{TR}=4000 \mathrm{~ms}$, echo time $(\mathrm{TE})=40 \mathrm{~ms}$ ) was used with the acquisition of each volume compressed into the first $1250 \mathrm{~ms}$ of the repetition time, creating a $2750 \mathrm{~ms}$ window in which participants could articulate a response in the absence of scanner noise. ${ }^{14}$ The other tasks (which did not involve speech) were studied using $\mathrm{TR}=2000 \mathrm{~ms}$ and $\mathrm{TE}=40 \mathrm{~ms}$. To facilitate anatomical localisation of activation, a high-resolution inversion recovery image data-set was also acquired, with $3 \mathrm{~mm}$ contiguous slices and an in-plane resolution of $3 \mathrm{~mm}$ ( $\mathrm{TR}=1600 \mathrm{~ms}$, inversion time $(\mathrm{TI})=180 \mathrm{~ms}, \mathrm{TE}=80 \mathrm{~ms}$ ).

\section{Cognitive tasks}

\section{$\mathrm{N}$-back memory task}

In all conditions participants were presented with a series of letters which they viewed using a prismatic mirror. The interstimulus interval was $2 \mathrm{~s}$. During the baseline (0-back) condition, individuals were required to move a joystick to the left when the letter ' $\mathrm{X}$ ' appeared. During the 1-back and 2-back conditions, participants were required to press a button on the joystick with their right index finger if the currently presented letter was the same as that presented one or two letters beforehand respectively. The three conditions were presented in 10 alternating $30 \mathrm{~s}$ blocks matched for the number of target letters per block (i.e. two or three), in pseudorandom order. Reaction time and the accuracy of the responses were recorded electronically by computer.

\section{Overt verbal fluency task}

Participants were required to say aloud a word beginning with a visually presented letter. The stimuli, each subtending an angle of $5^{\circ}$, were presented visually on a black screen, viewed through a mirror. Cognitive load was modulated with two levels of task difficulty, 'easy' and 'hard' conditions, which involved letters that differed with respect to the ease with which volunteers can usually generate words beginning with them. The 'easy' condition involved the letters L, T, C, P, S; the 'hard' condition: O, N, E, F, G. ${ }^{14}$ Incorrect responses were defined as words that were proper names, repetitions or grammatical variations of the previous word, and 'pass' responses. Letters were presented in $28 \mathrm{~s}$ blocks of seven stimuli at $4 \mathrm{~s}$ intervals. The control condition of word repetition comprised $28 \mathrm{~s}$ blocks of 7 presentations of the word 'rest' at $4 \mathrm{~s}$ intervals, which participants were required to read aloud. Five blocks of each condition (hard/easy/repetition) were presented in random order.

Verbal responses were recorded via an MRI-compatible microphone on Cool Edit 2000 for Windows. To ensure that participants heard their responses clearly, their speech was transmitted by an MRI-compatible microphone, amplified by a computer sound card and relayed back through an acoustic MRI sound system (Ward Ray, Hampton Court, UK), and noise-insulated, stereo headphones at a volume of 91 plus or minus $2 \mathrm{~dB}$.

\section{Image processing and analysis}

The data were realigned ${ }^{15}$ then smoothed using a Gaussian filter (full width half maximum $7.2 \mathrm{~mm}$ ). Responses to the experimental paradigms were detected by convolving each component of the design with each of two gamma variate functions (peak responses at 4 and $8 \mathrm{~s}$ respectively). The best fit between the weighted sum of these convolutions and the time series at each voxel was computed using the constrained blood oxygen level-dependent (BOLD) effect model. ${ }^{16}$ A goodness of fit statistic comprising the ratio of the sum of squares of deviations from the mean image intensity (over the whole time series) divided by the sum of squares of deviations due to the residuals (SSQ ratio) was then computed at each voxel.

The data were then permuted by a wavelet-based method ${ }^{17}$ to calculate the null distribution of SSQ ratios under the assumption of no experimentally determined response. This was used to calculate the critical value of SSQ ratio needed to threshold the maps at a type I error rate of $<1$. The detection of activated voxels was then extended from voxel to cluster level. ${ }^{18}$ To minimise the potential confounding effects of between-group and between-condition variation in task performance, in the analysis of data from the verbal fluency and $N$-back tasks the BOLD response in each person was modelled using only trials associated with correct responses.

In addition to the SSQ ratio, the size of the BOLD response to each experimental condition was computed for each individual at each voxel as a percentage of the mean resting image intensity level. In order to calculate the BOLD effect size, the difference between the maximum and minimum values of the fitted model for each condition was expressed as a percentage of the mean image intensity level over the whole time series.

Table 1 Age, IQ, gender and psychopathology ratings across groups

\begin{tabular}{|c|c|c|c|}
\hline Variable & Controls $(n=15)$ & At-risk group $(n=17)$ & Psychosis group ( $n=10)$ \\
\hline Age, years: mean (s.d.) & $25.4(4.9)$ & $24.2(4.1)$ & $25.5(5.9)$ \\
\hline Gender, male:female & $11: 4$ & $12: 5$ & $7: 3$ \\
\hline NART IQ: mean (s.d.) & $111.2(7.2)$ & $102.9(11.9)$ & $103.6(9.2)$ \\
\hline PANSS total: mean (s.d.) & N/A & 51. 9 (12.7) & $58.1(9.5)$ \\
\hline PANSS positive: mean (s.d.) & N/A & $11.7(3.4)$ & $18.5(4.6)$ \\
\hline PANSS negative: mean (s.d.) & N/A & $10.6(4.1)$ & $10.0(2.3)$ \\
\hline PANSS general: mean (s.d.) & N/A & $20.9(9.2)$ & $29.6(5.9)$ \\
\hline
\end{tabular}


The SSQ ratio maps for each individual were transformed into the standard space of Talairach and Tournoux ${ }^{19}$ using a two-stage warping procedure. ${ }^{20}$ Group activation maps were computed by determining the median SSQ ratio at each voxel (across all individuals) in the observed and permuted data maps. The distribution of median SSQ ratios from the permuted data was used to derive the null distribution of SSQ ratios and the critical SSQ ratio to threshold group activation maps at a cluster level threshold of $<1$ expected type I error cluster per brain.

Comparisons of responses between groups or experimental conditions was performed by fitting the data at each intracerebral voxel at which all participants had non-zero data using a linear model of the type:

$$
Y=a+b X+e
$$

where $Y$ is the vector of BOLD effect sizes for each individual, $X$ is the contrast matrix for the particular intercondition/group contrasts required, $a$ is the mean effect across all individuals in the various conditions/groups, $b$ is the computed group/condition difference and $e$ is a vector of residual errors. The model was fitted by minimising the sum of absolute deviations rather than the sums of squares to reduce outlier effects. The null distribution of $b$ was computed by permuting data between conditions/groups (assuming the null hypothesis of no effect of experimental condition or group membership) and refitting the above model.

In order to examine the data for a linear trend in activation across groups (controls, at-risk and psychosis) we carried out an orthogonal polynomial trend analysis in which the linear trend was coded as $-1,0,1$ (controls, at-risk and psychosis) and the orthogonal polynomial trend as $-1,2$ and -1 . Our hypothesis was that the linear trend would be significant but the quadratic trend would not be (i.e. there would be a linear trend but no significant departure from linearity). This would indicate that the order of responses would be controls $>$ at-risk $>$ psychosis or psychosis $>$ at-risk $>$ controls. This analysis was carried out using the effect size (beta) maps (which represented percentage changes in BOLD response) for each individual in each group after these had been transformed into standard space.

Voxel- and cluster-level maps of voxels and clusters showing significant linear and quadratic effects were computed using permutation testing as described above. The threshold for cluster-level analysis was chosen to give $<1$ false activated cluster per brain.

The method of analysis we employed (XBAM) uses median statistics to control outlier effects and permutation rather than normal theory-based inference. The main test statistic is computed by standardising for individual differences in residual noise before embarking on second-level, multiperson testing using robust permutation-based methods. Approaches using a mixed effects analysis, and permutation-based and cluster-level inference appear to be more valid than analyses involving simple random effects and voxel-level inference. ${ }^{21}$

\section{Results}

\section{Task performance}

In the $N$-back task there were no significant group differences in mean reaction time $(P=0.44)$, and no differences in the number of errors during the 1- and 2-back conditions $(P=0.49)$.

In the verbal fluency task there were no significant group differences in mean reaction time $(P=0.81)$. There was a group difference in the proportion of movements made to the right $(F=4.05$, d.f. $=2, P=0.028)$ : controls made more such movements than the at-risk group, with the psychosis group intermediate between them. There were no group differences in the number of errors produced during either the 'easy' $(P=0.45)$ or 'hard' versions of the verbal fluency task $(P=0.82)$.

\section{Regional activation}

$N$-back tasks, within-group activation (voxel $P<0.05$, cluster $P<0.01)$

1-back. In the control group, there was activation in the left inferior frontal gyrus and the posterior parietal cortex bilaterally. In the at-risk group, activation was evident in the inferior and middle frontal gyri bilaterally, the left inferior parietal and right inferior temporal cortex, and the left fusiform gyrus. The psychosis group displayed activation in the middle and superior frontal gyri bilaterally, the right inferior frontal gyrus, the left insula, the medial parietal cortex bilaterally, the right middle temporal gyrus and the right thalamus.

2-back. In the control group there was activation in the left precentral and medial frontal gyrus, the right inferior frontal gryus, and the left posterior and right medial parietal cortex. In the at-risk group, activation was evident in the right inferior frontal and the left middle frontal gyrus, and in the right posterior cortex and left precuneus. The psychosis group displayed activation in the inferior and middle frontal gyri bilaterally, the middle temporal gyrus bilaterally, and in the left thalamus and caudate.

\section{$\mathrm{N}$-back tasks, between-group differences in activation (voxel} $P<0.05$, cluster $P<0.01$ )

1-back. There was differential activation across the three groups in the left inferior parietal lobule and the right angular gyrus. In both these areas the at-risk group showed less activation than controls but more activation than the psychosis group (post hoc $t$-tests, $P<0.05)$ (Fig. 1 and Table 2).

2-back. Differential activation across the three groups was evident in the right insula and left inferior frontal gyrus, the right inferior parietal lobule, the left precuneus and right medial/ superior frontal gyrus. In each of these areas the at-risk group showed less activation than controls but more activation than the psychosis group (post hoc $t$-tests, $P<0.05$ ) (Fig. 1 and Table 3).

Verbal fluency task, within-group activation (voxel $P<0.05$, cluster $P<0.01)$

'Easy' condition. Controls showed activation in the left inferior and superior frontal gyri, the at-risk group activated the left inferior frontal and left fusiform gyri, right insula, and left superior frontal gyrus, and the psychosis group activated the left precentral gyrus, right insula, and the left inferior parietal and fusiform cortex.

'Hard' condition. Controls displayed activation in the left inferior frontal gyrus and inferior parietal lobule, and the right posterior cerebellar cortex. The at-risk group activated the left inferior frontal gyrus, the left superior frontal gyrus, and the psychosis group activated the left precentral gyrus and insula, and the right inferior frontal gyrus, insula and anterior cingulate gyrus. 

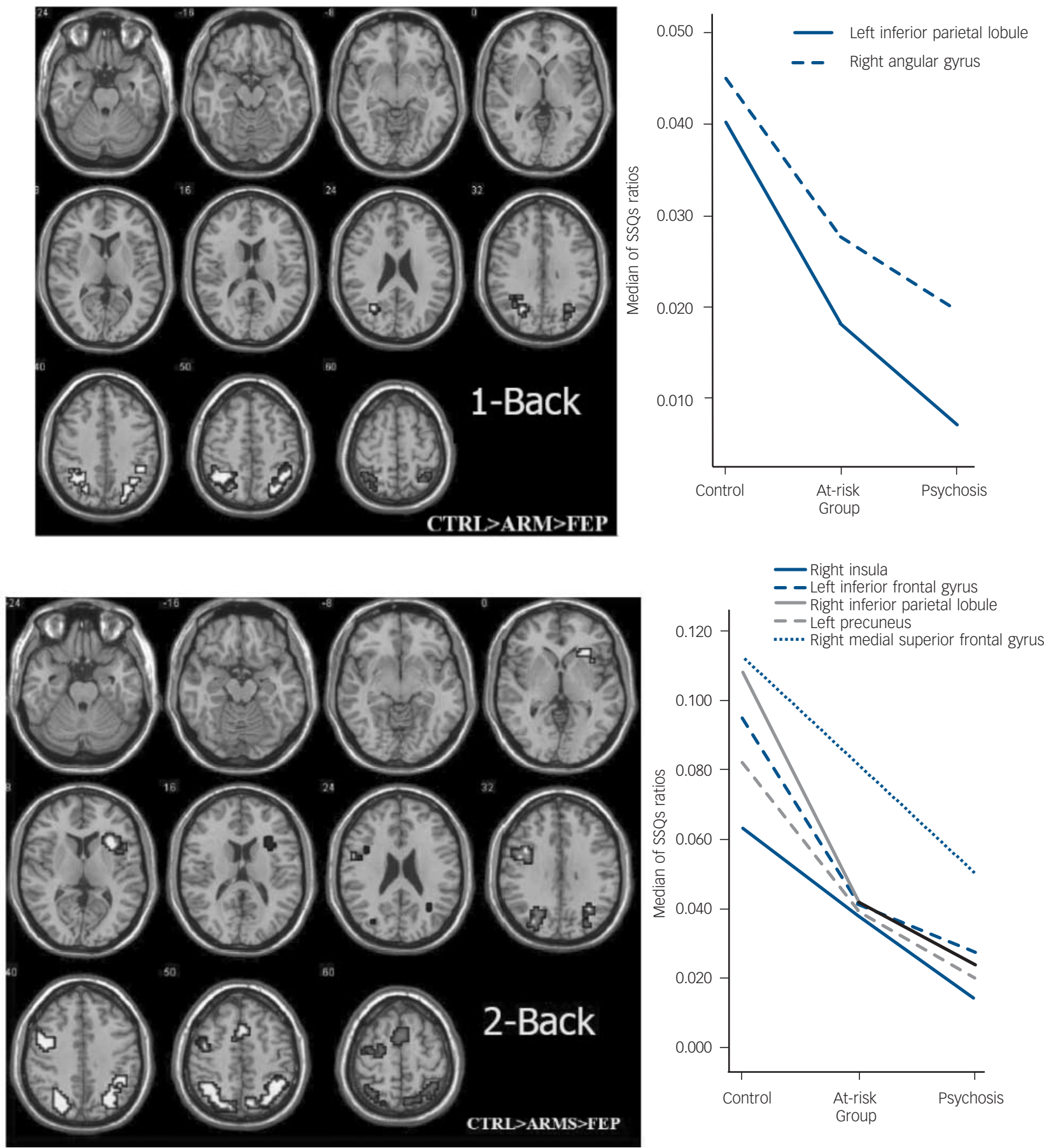

Fig. 1 Group differences in cluster activation during the 1-back and 2-back conditions of the $N$-back task. For the 1-back condition, activation was greatest in controls, weakest in the psychosis group and intermediate in the at-risk group in the left inferior parietal lobule and in the right angular gyrus. Differential activation during the 2-back condition was greatest in controls, weakest in the psychosis group and intermediate in the at-risk group in the lateral prefrontal, insular and parietal cortex, and in the precuneus, The left side of the brain is shown on the left of the figure (voxel $P<0.05$, cluster $P<0.01$ ). SSQRs, sum of squares of deviations due to the residuals.

Verbal fluency tasks between-group differences in activation (voxel $P<0.05$, cluster $P<0.01)$

'Easy' condition. There was differential activation across the three groups in a region which included both the opercular and dorsal parts of the left inferior frontal gyrus (Fig. 2 and Table 4). The at-risk group showed less activation in this region than controls but more activation than the psychosis group (post hoc $t$-tests, $P<0.05)$
'Hard' condition. Differential activation across the three groups was evident in a region which extended superiorly from the dorsal part of inferior frontal gyrus to adjacent middle frontal and precentral gyri (Fig. 3 and Table 5). In this region, the at-risk group showed less activation than the controls but greater activation than the psychosis group (post hoc $t$-tests, $P<0.05$ ).

The reverse pattern of differential activation was evident in a more ventral region focused on the left anterior insula. In this region activation was again intermediate in the at-risk group, 


\begin{tabular}{|llc|}
\hline $\begin{array}{l}\text { Table } 2 \\
\text { Talairach and Tournoux } \\
\text { coordinates }(x, y, z)\end{array}$ & Anatomical region & $\begin{array}{c}\text { Cluster size } \\
\text { (number of voxels) }\end{array}$ \\
\hline $32,-59,17$ & Posterior part of right middle temporal gyrus & 37 \\
\hline$-40,-48,37$ & Left inferior parietal lobule & 39 \\
\hline $29,-63,31$ & Right precuneus & 40 \\
\hline $40,-48,42$ & Right inferior parietal lobule & 7 \\
\hline$-22,-59,26$ & Left precuneus & 40 \\
\hline
\end{tabular}

but was greatest in the psychosis group and weakest in the controls (Fig. 3 and Table 6). Post hoc pairwise comparisons confirmed that in this region the at-risk group showed greater activation than controls, with a trend for less activation than the psychosis group ( $t$-tests, $P<0.05)$.

\section{Effects of medication}

Within the psychosis group (the only group which included participants who had received antipsychotic medication), there was no significant correlation (voxel $P<0.05$, cluster $P<0.01$ ) between activation in the regions that were differentially engaged across groups during each task and either the daily or cumulative dose (in chlorpromazine equivalents) of antipsychotic treatment, or the duration of antipsychotic treatment.

\section{Discussion}

The present study used functional MRI to study the neural substrate of executive functions and working memory in individuals with an at-risk mental state. The $N$-back task engages verbal working memory and requires the suppression of responses to currently presented stimuli. Verbal fluency entails the intrinsic generation of a verbal response, suppression of inappropriate responses and recalling and utilising previous responses.
In line with our hypothesis, there was a consistent pattern of differential activation across the groups for both tasks: during the $\mathrm{N}$-back and verbal fluency paradigms, the level of regional activation in the at-risk group was intermediate between that in the psychosis group and controls. This is the first study to demonstrate statistically intermediate patterns of activation in an at-risk group, compared with controls and participants with psychosis. These differences were evident in brain regions that are normally activated during these paradigms in volunteers: the prefrontal and parietal cortex during the $N$-back task, and the prefrontal and anterior cingulate cortex during verbal fluency. ${ }^{22-28}$ The differential activation was not attributable to impairments in task performance, as there were no significant differences in the speed or accuracy of responses across groups, and the analysis selectively modelled the BOLD response to those trials associated with correct responses. The lack of difference in behavioural performance allows the interpretation of activations to proceed knowing that the psychological task is being carried out to an equal level by all participants and hence, any remaining difference in activation is likely to be due to the disorder of interest, rather than a non-specific correlate of poor performance. The lack of behavioural difference is due both to excluding from the analysis individuals who perform the task very badly and to the study being powered to detect physiological changes, rather than neuropsychological differences, between the groups.
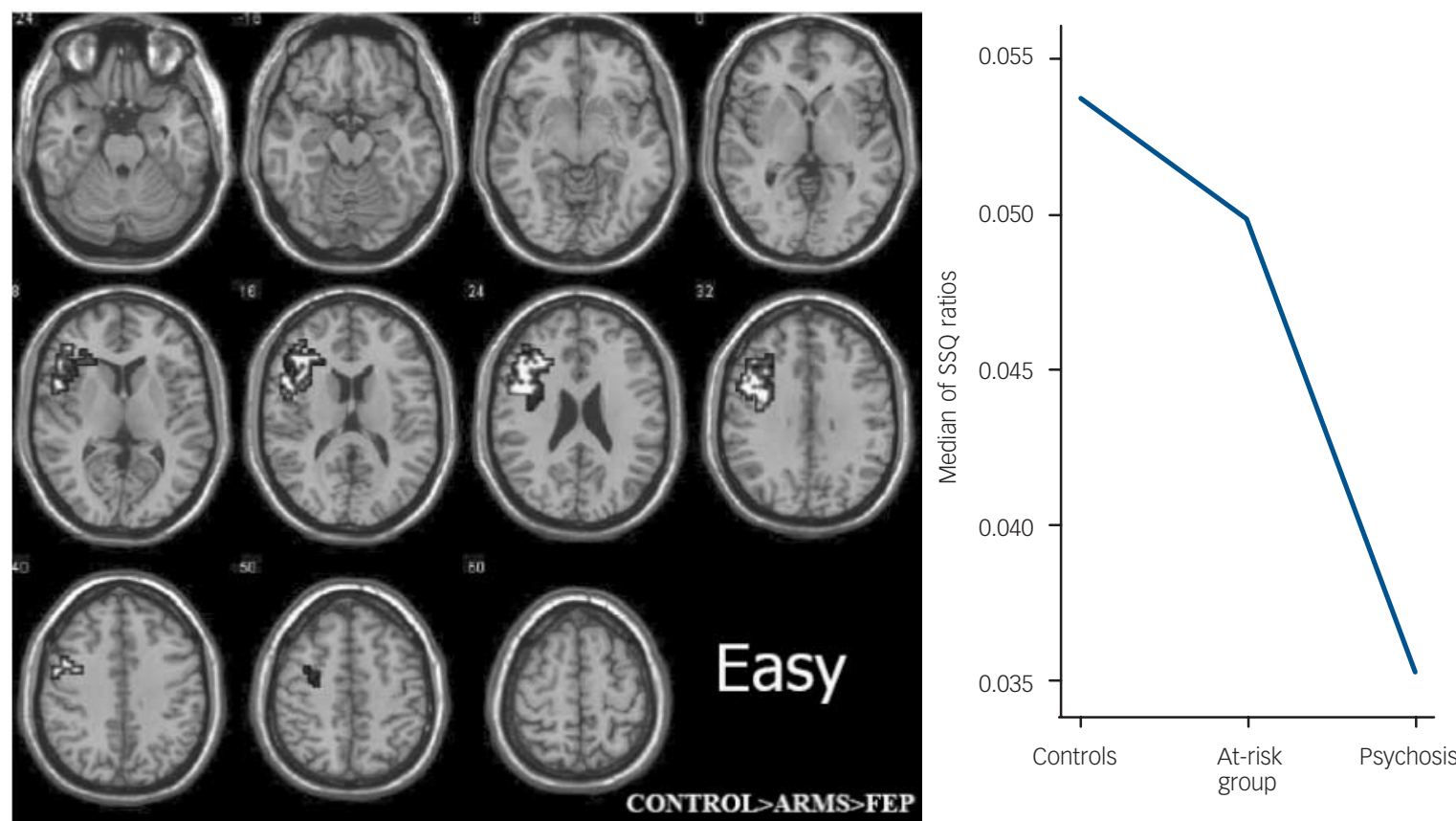

Fig. 2 Group differences in left inferior frontal cluster activation during 'easy' verbal fluency.

The at-risk group showed greater activation than the psychosis group but less than the controls. The left side of the brain is shown on the left of the figure (voxel $P<0.05$, cluster $P<0.01)$. SSQ ratio, sum of squares of deviations due to the residuals. 


\begin{tabular}{|c|c|c|c|}
\hline $\begin{array}{l}\text { Talairach and Tournoux } \\
\text { coordinates }(x, y, z)\end{array}$ & Anatomical region & Brodmann area & $\begin{array}{c}\text { Cluster size } \\
\text { (number of voxels) }\end{array}$ \\
\hline $40,-44,42$ & Right inferior parietal lobule & 40 & 38 \\
\hline$-22,-70,42$ & Left precuneus & 7 & 35 \\
\hline$-40,-41,37$ & Left inferior parietal lobule & 40 & 31 \\
\hline $29,-59,31$ & Right precuneus & 7 & 29 \\
\hline$-18,-74,17$ & Left calcarine sulcus & 17 & 26 \\
\hline $32,22,-2$ & Right post insula/claustrum & & 22 \\
\hline$-40,11,26$ & Left inferior frontal gyrus & 44 & 19 \\
\hline $4,11,48$ & Medial part right superior frontal gyrus & 6 & 18 \\
\hline $36,-56,48$ & Right superior parietal lobule & 7 & 15 \\
\hline $0,15,42$ & Anterior cingulate & 32 & 12 \\
\hline$-22,-4,48$ & Left superior frontal gyrus & 6 & 12 \\
\hline
\end{tabular}

\section{Table 4 Controls < at-risk < psychosis: 'easy' verbal fluency task between-group differences in activation}

\begin{tabular}{llcc}
$\begin{array}{l}\text { Talairach and Tournoux } \\
\text { coordinates }(x, y, z)\end{array}$ & Anatomical region & Brodmann area & $\begin{array}{c}\text { Cluster size } \\
\text { (number of voxels) }\end{array}$ \\
$-36,30,15$ & Left inferior frontal gyrus.(anterior portion) & 45 & 36 \\
\hline$-40,7,20$ & Left inferior frontal gyrus (dorsal portion) & 44 & 34 \\
\hline$-47,11,9$ & Left inferior frontal gyrus (frontal operculum) & 44 & 26 \\
\hline
\end{tabular}

\begin{tabular}{|c|c|c|c|}
\hline $\begin{array}{l}\text { Talairach and Tournoux } \\
\text { coordinates }(x, y, z)\end{array}$ & Anatomical region & Brodmann area & $\begin{array}{c}\text { Cluster size } \\
\text { (number of voxels) }\end{array}$ \\
\hline$-43,11,15$ & Left inferior Frontal gyrus (frontal operculum). & 44 & 18 \\
\hline
\end{tabular}

\begin{tabular}{|c|c|c|c|}
\hline $\begin{array}{l}\text { Talairach and Tournoux } \\
\text { coordinates }(x, y, z)\end{array}$ & Anatomical region & Brodmann area & $\begin{array}{c}\text { Cluster size } \\
\text { (number of voxels) }\end{array}$ \\
\hline$-32,15,-2$ & Left anterior insula & 47 & 24 \\
\hline
\end{tabular}

Similarly, the findings are unlikely to be related to effects of antipsychotic medication as both the at-risk group and controls were medication naïve, and in the psychosis group there was no relationship between medication exposure and activation in the regions that were differentially engaged across groups. Further, when quadratic trend analysis was carried out, there were no significant clusters activated differentially across the groups: again, this indicates that there was a predominantly linear relationship in activation across the groups on all tasks.

The brain regions where we observed differential activation in the at-risk group correspond to those that have previously been reported as sites of abnormal activation in functional imaging studies of schizophrenia. Thus, patients with schiziophrenia show reduced activation in the prefrontal and parietal cortex during the $\mathrm{N}$-back task, ${ }^{24}$ in the parietal cortex during random movement generation, ${ }^{29}$ and in the left prefrontal cortex during verbal fluency. ${ }^{30}$ There has only been one previous functional imaging study involving participants with an at-risk mental state. This reported differential prefrontal activation during a visual oddball paradigm in an at-risk group relative to controls and patients with schizophrenia. ${ }^{6}$

During the 1-back condition of the $\mathrm{N}$-back task, the at-risk group showed attenuated activation in the parietal cortex relative to controls. These differences became more extensive during the more demanding 2-back condition, and were accompanied by additional reductions in prefrontal activation. Nevertheless, the magnitude of activation in the at-risk group remained intermediate to that in the control and psychosis groups when the task demands were increased. Similarly, although during the 'hard' verbal fluency task the pattern of activation differences in the insula was reversed (discussed further below), the magnitude of activation in the at-risk group remained intermediate relative to that in the other groups, as during the 'easy' version of the task, and did not more closely resemble that in the psychosis group.

During the 'hard' verbal fluency task, engagement of the left insula was greatest in the psychosis group, intermediate in the at-risk group and weakest in controls. In the dorsal part of the left inferior frontal gyrus the opposite applied, with greatest activation in controls and least in the psychosis group. Relatively greater engagement of the insula in the psychosis group in the context of increased demands on controlled word retrieval ${ }^{31}$ and selection among competing words ${ }^{32}$ might reflect a compensatory response in the group in whom processing was most compromised and who showed the weakest engagement of the inferior frontal gyrus.

The overall pattern of the findings is consistent with data from neuropsychological studies of the at-risk mental state. These indicate that individuals who are at risk display impairments on tasks of executive functions and memory (including $\mathrm{N}$-back and 

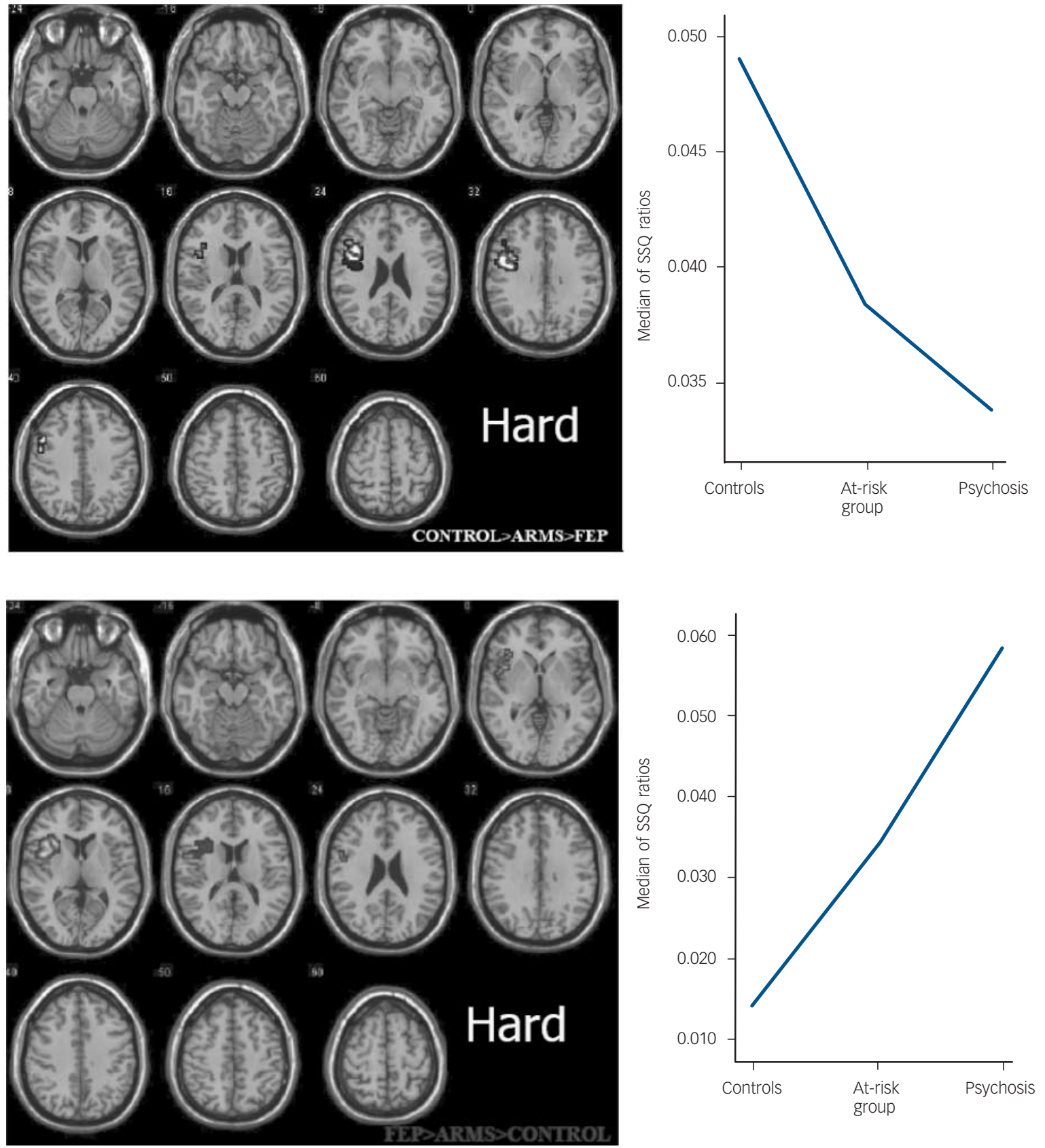

Fig. 3 Group difference in cluster activation during 'hard' verbal fluency.

When the task demands were high, there was a differential engagement of dorsolateral prefrontal cortex with activation greatest in the control group, weakest in the psychosis group, and intermediate in the at-risk group. However, on the same version of the task, there was differential engagement of the left anterior insula. When task demands were high activation in this region was greatest in the psychosis group, weakest in the controls and intermediate in the at-risk group. The left side of the brain is shown on the left of the figure (voxel $P<0.05$, cluster $P<0.01$ ). SSQRS, sum of squares of deviations due to the residuals.

verbal fluency) that are qualitatively similar, but less severe, than those evident in patients with schizophrenia. ${ }^{33-38}$ Similarly, structural MRI studies suggest that the at-risk mental state is associated with reductions in grey-matter volume in similar regions that show volume reductions in schizophrenia, including the inferior frontal, cingulate and temporal cortex. ${ }^{5,39}$

As the at-risk group had a high risk of developing a psychotic disorder but did not have psychosis, the functional abnormalities they displayed can be seen as a correlate of their increased vulnerability to psychosis. It is unlikely that the findings reflected the erroneous inclusion of individuals who already had psychosis, or who were already progressing towards schizophrenia, as inclusion required detailed assessment by at least two clinicians experienced in the management of the at-risk mental state. In addition, participants were closely monitored for signs of frank psychosis subsequent to scanning.

\section{Limitations of the study}

This study reports cross-sectional data on three groups: those at risk of developing a psychotic disorder, those with psychosis and controls. As noted above, the findings in the at-risk group may be a correlate of their increased vulnerability to psychosis. However, to determine this formally will require a longitudinal 
study: a study informed by the findings presented here and in particular whether the pattern and degree of activation during executive and working memory tasks predicts transition to psychosis in a clinical high-risk group.

\section{Conclusions}

The at-risk mental state is associated with abnormalities of regional brain function that are qualitatively similar but less severe than those seen in patients who have just developed schizophrenia. These may underlie the impairments in executive function and working memory that are evident in this group and can be seen as correlates of their increased vulnerability to psychosis.

Matthew R. Broome, BSC, PhD, MBChB, MRCPsych, Section of Neuroimaging, Division of Psychological Medicine, Institute of Psychiatry, King's College London, and Health Sciences Research Institute, Warwick Medical School, University of Warwick, Coventry, UK; Pall Matthiasson, MD, MRCPsych, PhD, section of Neuroimaging Division of Psychological Medicine, Institute of Psychiatry, King's College London; Paolo Fusar-Poli, MD, Section of Neuroimaging, Division of Psychological Medicine, Institute of Psychiatry, King's College London, UK, and Department of Applied and Psychobehavioural Health Sciences, University of Pavia, Italy; James B. Woolley, BSC, MBBS, MRCP, MRCPsych, Louise C. Johns, DPhil, DClinPsy, Paul Tabraham, BSC, DClinPsy, Elvira Bramon, MD, PhD, Section of Neuroimaging, Division of Psychological Medicine, Institute of Psychiatry, King's College London, UK Lucia Valmaggia, PhD, DClinPsy, Section of Neuroimaging, Division of Psychological Medicine, Institute of Psychiatry, King's College London, UK, and Department of Psychiatry and Neuropsychology, Maastricht University, Maastricht, The Netherlands; Steven C. R. Williams, PhD, Neuroimaging Research Group, Department of Neurology, Institute of Psychiatry, King's College London, UK; Michael J. Brammer $\mathrm{PhD}$, Xavier Chitnis, MSC, Brain Image Analysis Unit, Department of Biostatistics and Computing, Institute of Psychiatry, King's College London, UK; Philip K. McGuire, $\mathrm{MD}$, PhD, FRCPsych, Section of Neuroimaging, Division of Psychological Medicine, Institute of Psychiatry, King's College London, UK.

Correspondence: Matthew R. Broome, Warwick Medical School, University of Warwick, Gibbet Hill, Coventry CV4 7AL, UK. Email: m.r.broome@warwick.ac.uk

\section{Acknowledgements}

OASIS is supported by the Guy's and St Thomas' Charitable Foundation and the South London and Maudsley NHS Trust. E.B. is a Wellcome research fellow. Thanks go to all the clients, staff and referrers of both OASIS and Lambeth Early Onset Services. The authors are grateful to Dr Paul Allen for advice on interpretation of the verbal fluency data.

\section{References}

1 Miller TJ, McGlashan TH, Rosen JL, Somjee L, Markovich PJ, Stein K, et al. Prospective diagnosis of the initial prodrome for schizophrenia based on the Structured Interview for Prodromal Syndromes: preliminary evidence of interrater reliability and predictive validity. Am J Psychiatry 2002; 159: 863-5.

2 Yung AR, Phillips LJ, Yuen HP, Francey SM, McFarlane CA, Hallgren M, et al. Psychosis prediction: 12-month follow up of a high-risk ('prodromal') group. Schizophr Res 2003; 60: 21-32.

3 Wagner M, Frommann I, Jessen F, Pukrop R, Bechdolf A, Ruhrmann S, et al. Cognitive and neurobiological risk indicators in early and late prodromal stages. Schizophr Res 2006; 86 (suppl): s35-6.

4 Pukrop R, Ruhrmann S, Schultze-Lutter F, Bechdolf A, Brockhaus-Dumke A Klosterkotter J. Neurocognitive indicators for a conversion to psychosis: Comparison of patients in a potentially initial prodromal state who did or did not convert to a psychosis. Schizophr Res 2007; 92: 116-25.

5 Pantelis C, Velakoulis D, McGorry PD, Wood SJ, Suckling J, Phillips L, et al. Neuroanatomical abnormalities before and after onset of psychosis: a crosssectional and longitudinal MRI comparison. Lancet 2003; 361: 281-8.

6 Morey RA, Inan S, Mitchell TV, Perkins DO, Lieberman JA, Belger A. Imaging frontostriatal function in ultra-high-risk, early, and chronic schizophrenia during executive processing. Arch Gen Psychiatry 2005; 62: 254-62.

7 Yung AR, Phillips L, McGorry PD, McFarlane CA, Francey S, Harrigan S, et al. Prediction of psychosis. A step towards indicated prevention of schizophrenia. Br J Psychiatry 1998; 172 (suppl 33): s14-20.

8 Broome MR, Woolley JB, Johns LC, Valmaggia LR, Tabraham P, Gafoor R, et al Outreach and Support in South London (OASIS): implementation of a clinical service for prodromal psychosis and the at risk mental state. Eur Psychiatry 2005; 20: 372-8.

9 World Health Organization. ICD-10: The ICD-10 Classification of Mental and Behavioural Disorders: Clinical Descriptions and Diagnostic Guidelines. WHO, 1992.

10 McGuffin P, Farmer A, Harvey I. A polydiagnostic application of operational criteria in studies of psychotic illness. Development and reliability of the OPCRIT system. Arch Gen Psychiatry 1991; 48: 764-70.

11 American Psychiatric Association. Diagnostic and Statistical Manual of Mental Disorders (4th edn) (DSM-IV). APA, 1994.

12 Nelson HE. National Adult Reading Test (NART) Manual. nferNelson, 1982.

13 Kay S, Fiszbein A, Opler L. The positive and negative symptom scale (PANSS) for schizophrenia. Schizophr Bull 1987; 13: 261-76.

14 Fu CH, Morgan K, Suckling J, Williams SC, Andrew C, vythelingum GN, et al. A functional magnetic resonance imaging study of overt letter verbal fluency using a clustered acquisition sequence: greater anterior cingulate activation with increased task demand. Neuroimage 2002; 17: 871-9.

15 Bullmore ET, Brammer MJ, Rabe-Hesketh S, Curtis VA, Morris RG, Williams SC, et al. Methods for diagnosis and treatment of stimulus-correlated motion in generic brain activation studies using fMRI. Hum Brain Mapp 1999; 7: 38-48.

16 Friman $\mathrm{O}$, Borga $\mathrm{P}$, Lundberg $\mathrm{P}$, Knutsson $\mathrm{H}$. Adaptive analysis of fMRI data. Neuroimage 2003; 19: 837-45.

17 Bullmore ET, Long C, Suckling J, Fadili J, Calvert G, Zelaya F, et al. Coloured noise and computational inference in neurophysiological (fMRI) time series analysis. Resampling methods in time and wavelet domains. Hum Brain Mapp 2001; 12: 61-78.

18 Bullmore ET, Suckling J, Overmayer S, Rabe-Hesketh S, Taylor E, Brammer MJ. Global, voxel and cluster tests, by theory and permutation, for a difference between two groups of structural MR images of the brain. IEEE Trans Med Imaging 1999; 18: 32-42.

19 Talairach J, Tournoux P. Co-Planar Stereotaxic Atlas of the Human Brain 3-Dimensional Proportional System: An Approach to Cerebral Imaging. Thieme Publishing Group, 1988.

20 Brammer MJ, Bullmore ET, Simmons A, Williams SCR, Grasby PM, Howard RJ, et al. Generic brain activation mapping in fMRI: a nonparametric approach. Magn Reson Imaging 1997; 15: 763-70.

21 Thirion B, Pinel P, Meriaux S, Roche A, Dehaene S, Poline J-B. Analysis of a large fMRI cohort: statistical and methodological issues for group analysis. Neuroimage 2007; 35: 105-20.

22 Callicott JH, Egan MF, Mattay VS, Bertolino A, Bone AD, Verchinksi B, et al. Abnormal fMRI response of the dorsolateral prefrontal cortex in cognitively intact siblings of patients with schizophrenia. Am J Psychiatry 2003; 160: 709-19.

23 Callicott JH, Mattay VS, Verchinski BA, Marenco S, Egan MF, Weinberger DR. Complexity of prefrontal cortical dysfunction in schizophrenia: more than up or down. Am J Psychiatry 2003; 160: 2209-15.

24 Honey R, Honey G, O'Loughlin C, Sharar SR, Kumaran D, Bullmore ET, et al. Acute ketamine administration alters the brain responses to executive demands in a verbal working memory task: an fMRI study. Neuropsychopharmacology 2004; 29: 1203-14.

25 Crespo-Facorro B, Paradiso S, Andreasen N, O'Leary DS, Watkins GL, Boles Ponto LL, et al. Recalling word lists reveals 'cognitive dysmetria' in schizophrenia: a positron emission tomography study. Am J Psychiatry 1999; 156: 386-92.

26 Curtis V, Dixon T, Morris R, Bullmore ET, Brammer MJ, Williams SC, et al. Differential frontal activation in schizophrenia and bipolar illness during verbal fluency. J Affect Disord 2001; 66: 111-21.

27 Fu CH, suckling J, Williams SC, Andrew CM, Vythelingum GN, McGuire PK. Effects of psychotic state and task demand on prefrontal function in schizophrenia: an fMRI study of overt verbal fluency. Am J Psychiatry 2005; 162: 485-94.

28 Yurgelun-Todd D, Waternaux C, Cohen B, Gruber S, English C, Renshaw P. Functional magnetic resonance imaging of schizophrenic patients during word production. Am J Psychiatry 1996; 153: 200-5.

29 Spence SA, Brooks DJ, Hirsch SR, Liddle PF, Meehan J, Grasby PM. A PET study of voluntary movement in schizophrenic patients experiencing passivity phenomena (delusions of alien control). Brain 1997; 120 (Pt 11): 1997-2011.

30 Curtis V, Bullmore ET, Brammer MJ, Wright IC, Williams SC, Morris RG, et al. Attenuated frontal activation during a verbal fluency task in patients with schizophrenia. Am J Psychiatry 1998; 155: 1056-63.

31 Wagner AD, Pare-Blagoev EJ, Clark J, Poldrack RA. Recovering meaning: left prefrontal cortex guides controlled semantic retrieval. Neuron 2001; 31: 329-38. 
32 Moss HE, Abdallah S, Fletcher P, Bright P, Pilgrim L, Acres K, et al. Selecting among competing alternatives: selection and retrieval in the left inferior frontal gyrus. Cereb Cortex 2005; 15: 1723-35.

33 Brewer WJ, Francey SM, Wood SJ, Jackson HJ, Pantelis C, Phillips $\sqcup$, et al Memory impairments identified in people at ultra-high risk for psychosis who later develop first-episode psychosis. Am J Psychiatry 2005; 162: 71-8.

34 Hawkins KA, Addington J, Keefe RS, Christensen B, Perkins DO, Zipurksy R, et al. Neuropsychological status of subjects at high risk for a first episode of psychosis. Schizophr Res 2004; 67: 115-22.

35 Gschwandtner U, Aston J, Borgwardt S, Drewe M, Feinendegen C, Lacher D et al. Neuropsychological and neurophysiological findings in individuals suspected to be at risk for schizophrenia: preliminary results from the Base early detection of psychosis study - Fruherkennung von Psychosen (FEPSY). Acta Psychiatr Scand 2003; 108: 152-5.
36 Pflueger MO, Gschwandtner U, Aston J, Berger G, Borgwardt S, Drewe M, et al. Cognitive capability of individuals at risk with and without transition to psychosis. Eur Psychiatry 2007; 22: s30.

37 Broome MR, Johns LC, Valli I, Woolley JB, Tabraham P, Brett C, et al. Delusion formation and reasoning biases in those at clinical high risk for psychosis. Br J Psychiatry 2007; 51: s38-42.

38 Broome MR, Woolley JB, Tabraham P, Johns LC, Bramon E, Murray GK, et al. What causes the onset of psychosis? Schizophr Res 2005; 79: 23-34.

39 Borgwardt S, Riecher-Rössler A, Dazzan P, Chitnis X, Aston J, Drewe M, et al. Regional gray matter volume abnormalities in the at risk mental state. Biol Psychiatry 2007; 61: 1148-56.
Psychiatry

\section{Was the scoundrel (belial) of the Book of Proverbs a psychopath?}

\section{George Stein}

The first descriptions of psychopathy in the UK are attributed to Pritchard in 1835 and to Pinel in 1790 in France, but psychopathic characters have already appeared in the Bible. The Book of Proverbs includes a short epigram 6:12-19 of a person called the Scoundrel (belial):

Chapter 6,

12 'A scoundrel and a villain goes around with crooked speech winking the eyes,

13 shuffling the feet and pointing the fingers

14 with perverted mind devising evil and continually sowing discord.

15 On such a one calamity will descend suddenly in a moment damage beyond repair.

16 There are six things the lord hates and seven are an abomination to him.

17 Haughty eyes and a lying tongue and hands that shed innocent blood.

18 A heart that devises wicked plans, feet that hurry to run to evil.

19 A lying witness who testifies falsely and one who sows discord in the family.'

McKane, a professor of theology, wrote that the 'eesh belial (the scoundrel) was a destructive man rather than a worthless man. There is a deep seated malevolence... He is obsessed with the thought of hurting his fellows. He digs mischief and his words have the destructive fury of a blazing fire' (Proverbs: A New Approach; 1970, SCM Press). This is a reasonable description of an aggressive psychopath based on the biblical account of the belial rather than any modern clinical text

Most of the DSM-IV features of antisocial personality disorder are contained here. The rabbis interpreted the word belial to mean beli, without and Ya-al, the yoke, which means somebody living without the yoke of the Torah (the sacred Jewish law). This is not too far from the DSM-IV criteria A of antisocial personality disorder - 'pervasive disregard for and violation of the rights of others'. Other DSM-IV criteria for this personality disorder include failure to conform to social norms (v. 17 and 19); deceitfulness (v. 19), impulsivity (v. 18), irritability and aggression (v. 17), reckless disregard for safety of self or others (v. 15, a reference to capital punishment), and lack of remorse (v. 19, which probably also describes wife or family abuse). Possibly up to five out of the seven criteria for DSM-IV antisocial personality disorder are mentioned or alluded to in this Biblical text. This poem is actually shorter (93 words) than the DSM-IV summary of antisocial personality disorder (127 words). Psychopathic personality disorders must have been a major problem in ancient Israelite society if it was so well described and appeared in the Bible. 